\title{
PET/MRI: a new era in multimodality molecular imaging
}

\author{
Osman Ratib
}

Received: 22 October 2012/ Accepted: 10 January 2013/Published online: 12 February 2013

(C) Italian Association of Nuclear Medicine and Molecular Imaging 2013

\begin{abstract}
Hybrid PET/MR imaging has long been considered an impossible combination due to the difficulty of ensuring the functioning of PET detectors within the magnetic field of an MR scanner, and the artifacts from the electronics of the PET scanner, which can affect MR images. Recent advances in technology have brought to the market hybrid devices in which the two modalities are set side by side along a single bed or in which solid-state detector rings are integrated into the MRI gantry. While the clinical and added value of such hybrid devices is still to be demonstrated, the ability to acquire perfectly registered images from both modalities in a single imaging session offers tangible advantages to patients needing to undergo both examinations as part of their clinical workup. Such cases include patients with head and neck cancers, prostate cancers, patients undergoing staging, and monitoring of extensive and high-risk breast cancers and gynecological cancers, and pediatric oncology cases where repeated studies are required and the reduction of radiation exposure by replacing the CT component of PET/CT scans with MRI is particularly important. It is anticipated that wider adoption of PET/MR imaging will occur once the added value and improvement in diagnostic accuracy have been demonstrated in prospective clinical studies. The introduction of new PET tracers is also likely to lead to wider clinical use of PET/MR. One example is the recent
\end{abstract}

O. Ratib

Department of Medical Imaging and Information Sciences, University Hospital of Geneva, Geneva, Switzerland

O. Ratib ( $\square)$

Division of Nuclear Medicine and Molecular Imaging, University Hospital of Geneva, 24, rue Micheli-du-Crest, 1205 Geneva, Switzerland

e-mail: osman.ratib@hcuge.ch introduction of ${ }^{18} \mathrm{~F}$-labeled $\mathrm{NaF}$ for bone metastases that makes PET in combination with MR imaging ideal for the characterization of bone lesions. The development of other new tracers may extend the use of PET/MR to domains other than oncology, such as cardiology and neurology.

Keywords PET/MRI - Hybrid imaging · Nuclear medicine $\cdot$ Molecular imaging

\section{Introduction}

In recent years, hybrid PET/CT has gained wide acceptance as an improved imaging modality compared with PET or CT alone in tumor staging and the assessment of therapeutic response in numerous oncology applications [1, 2]. Several studies have reported significant improvements in diagnostic accuracy and lesion localization of the hybrid imaging modality compared both with the two imaging modalities alone and with the two imaging modalities viewed side by side $[3,4]$. Because it offers simultaneous assessment of metabolic function and anatomy, hybrid PET/CT has become one of the main imaging tools in clinical oncology, in some domains surpassing CT and PET alone. Accurate alignment of both modalities and the use of image fusion have clearly improved diagnostic accuracy and more importantly the confidence and conclusiveness of image interpretation reports compared with the degree of uncertainty that can occur in interpretation of each modality singly.

Along similar lines, advanced MRI techniques can provide precious complementary diagnostic information for soft tissue analysis, tumor detection, tissue characterization, and functional imaging. It is not uncommon today for an oncology patient to undergo both a PET/CT and an MRI scan, to obtain complementary diagnostic data. In some 
cases, the diagnostic value of MRI for the detection and staging of cancers can be superior to that of CT studies, which limits the value of the diagnostic CT studies that can be performed in combination with the PET/CT study to that of a simple anatomical localization and attenuation correction scan. It is therefore quite rational to anticipate that in such cases a hybrid PET/MRI scan could be more valuable than a PET/CT scan followed by a complementary MRI scan [5]. In addition, whole-body MRI has been reported to show excellent diagnostic performance by itself, and proposed as an alternative to or replacement for PET/CT [6]. Whole-body MRI, especially diffusion-weighted sequences that showed high sensitivity, are increasingly being used in oncology [7] for initial diagnosis or staging, assessment of metastases, staging of pregnant woman and children, and follow-up of therapeutic response.

The main limitation of MRI today is the length of imaging protocols and the number of imaging sequences required for a given diagnostic task. Unlike multi-detector scanners that can perform high-resolution whole-body acquisitions of full 3D data in a few seconds, MRI scans can, in reasonable acquisition times, still acquire only limited anatomical regions in a limited number of slices. A recent development allows volumetric acquisitions of highresolution 3D data without changes in coils or patient repositioning, but these are still limited in routine practice and require relatively long acquisition times.

In the recent generation of high-field MR scanners, however, the use of acquisition acceleration techniques, such as parallel imaging, have made both whole-body short time inversion recovery imaging and T1-weighted imaging possible in under $15 \mathrm{~min}$ of scanning time for each modality [6]. However, some compromises in spatial resolution and axial slice thickness have to be made in order to achieve reasonable acquisition times. Fast spin-echo imaging techniques allow detailed tissue contrast even in lung parenchyma and hilar structures with reduced blurring and signal loss. On the basis of these new imaging techniques, a whole-body hybrid imaging protocol combining a PET and MR study could be envisaged. Fast T1-weighted imaging techniques allow the generation of whole-body volume images in clinically acceptable imaging times. These images provide adequate information for tissue segmentation and generation of model-based attenuation correction maps. To a certain degree, they can also provide adequate localization images similar to low-dose wholebody CT images used in PET/CT.

\section{Hybrid PET/MRI systems}

Combining MRI with PET in a single device has always been a challenge and even considered impossible due to the interference between the MR magnetic field and PET detectors, consisting of photomultipliers. MR scanners are also quite sensitive to interference from electronic devices that, if placed too close to the center of the magnet, can generate artifacts in the MR images. The idea of combining these two imaging modalities is not new, however, and even predates the idea of combining a PET and a CT scanner. Some of the first work in this field was done in the early 1990s by Simon Cherry and Paul Marsden [8], who attempted to develop detectors that can be inserted in a magnetic field.

More recently, the development of solid-state detectors has opened a new perspective on the design of hybrid PET/ MR scanners.

In the current state of the art, several alternative designs are being explored in an attempt to provide coregistered PET and MR studies (Fig. 1). An early generation of avalanche photodiodes (APDs) was used to design a prototype PET insert dedicated to brain imaging for use in a whole-body MR scanner. Although the potential clinical application of this solution was limited due to its size, it paved the way for more advanced hybrid scanners featuring larger PET rings that were recently released on the market. Current APD detectors still have some technical limitations in terms of performance and acquisition, which does not allow time-of-flight (TOF) detection for more accurate localization of positron annihilation events. A future generation of solid-state detectors based on silicone photomultipliers will certainly soon become more widely available, providing an advantageous replacement for current detectors but at a higher cost.

However, until such time as solid-state technology is available for large-bore whole-body scanners, alternative solutions allowing sequential imaging are being explored [9]. The first prototype launched for clinical evaluation combines a conventional 3T MRI scanner (Achieva 3T X-series MRI system from Philips) with a TOF PET scanner (Gemini TF PET), positioned at each end of a common sliding bed, and allows $180^{\circ}$ rotation of the patient from one scanner to the other and accurate registration between the two modalities. The PET gantry is designed to provide magnetic shielding of photomultipliers so that they operate in nominal magnetic flux levels. Additional modifications allowed all the electronics to be moved from the PET gantry to the equipment room so as to satisfy the strict noise requirements of MRI. PET modifications were made to ensure no compromise in system performance in either PET or MRI.

While this alternative does not allow simultaneous acquisition of the two modalities, it resembles, in its design, current PET/CT systems where the patient is moved from one scanner to the other scanner, positioned 

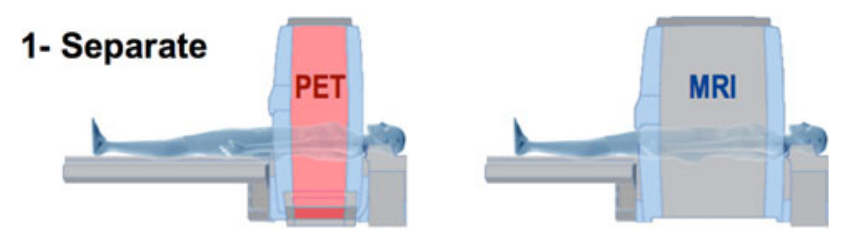

\section{2- Co-planar}

\section{3- Integrated}
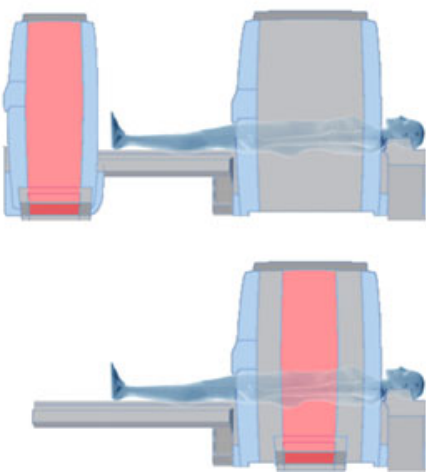

Fig. 1 Three different configurations of hybrid PET/MR imaging devices are available today: 1 two separate systems with the capacity for software registration of images. Some recent systems have a mobile table: in this way, the patient, without being moved from the table, can be transferred from one scanner to the other; 2 co-planar systems where a common bed moves the patient from one scanner to the other in a similar way to current PET/CT scanners; and 3 integrated systems which allow simultaneous acquisition of images from both modalities [25]

adjacent to it. Provided that the patient does not move between imaging studies, this solution allows sequential acquisition of co-registered PET and MR sequences.

Similarly, a simpler design by General Electric is based on two scanners located in separate rooms and a common table which allows patients, without being moved from the table, to be shuttled from one scanner to the other, thereby allowing subsequent registrations of images acquired sequentially in the two devices. This alternative, which is a little more challenging from the point of view of the logistics of moving patients between scanners, may be more cost-effective in a busy department where the two scanners could be used simultaneously for two different patients.

The first integrated whole-body system was launched recently by Siemens Medical Systems (mMR scanner). It integrates full-ring solid-state APD detectors in the gantry of a 3T MR scanner. This system allows simultaneous acquisition of PET and MRI images, which potentially reduces the total study time. The only limitation remains the low temporal performance of APD detectors with inability to provide TOF detection.

The remaining challenge for all hybrid PET/MR systems is the calculation of attenuation correction maps similar to those calculated from whole-body CT scans in hybrid PET/ CT devices. There have been several investigations on MRbased attenuation correction, but the emphasis has been mainly on MR image segmentation for derivation of an attenuation map [10-13]. Besides image segmentation, other technical challenges for effective attenuation correction in a whole-body PET/MR, including compensation for MR image truncation and correction for RF coils and accessories, also need to be met (Fig. 2).

\section{Clinical perspectives}

The first whole-body hybrid scanners, currently available in clinical settings, allowed exploration and evaluation of diagnostic effectiveness with the possible added value of acquiring both MR and PET studies on the same device with perfect registration of images from both modalities. Today, the first patients to benefit from such systems are those who need a PET scan and a complementary MRI scan in their clinical workup. Some of the most common applications for these joint diagnostic procedures are in oncology, and in particular in prostate cancer where most patients will undergo a diagnostic and follow-up MRI together with a PET/CT. Other applications include head and neck cancers which require MRI and PET not only for staging but also for follow-up for detection of recurrence after surgery and after treatments. Breast imaging is likely to see a growth of demand for hybrid PET/MR, given that today most diagnostic imaging and breast cancer screening includes an MRI scan; furthermore, a subset of patients submitted to complementary PET/CT could benefit from a hybrid PET/MR, thereby reducing unnecessary radiation from the CT component of the PET/CT scan. Along the same lines, PET/MR will be preferred over PET/CT in pediatric patients, in whom efforts to reduce radiation exposure are most critical.

It is, however, expected that broader applications of hybrid PET/MR will be developed in the near future as ongoing clinical validations are underway in numerous centers with a variety of new applications. We are observing an increasing demand for PET/MR studies in patients with gynecological cancers, for example, in whom MRI is the modality of choice for diagnosis and staging, although a subset of patients with extensive lesions can benefit from PET studies for more accurate identification of distant lymph node metastases. In such patients referring physicians are often reluctant to order a PET/CT, preferring to avoid unnecessary radiation exposure from the CT component over the pelvic area in young women. The combination of PET and MR modalities offers a competitive alternative to PET/CT in these patients. Prospective studies are still necessary to define the selection criteria of patients that could benefit from such studies, deriving welldemonstrated added value in diagnostic accuracy and patient outcome. 


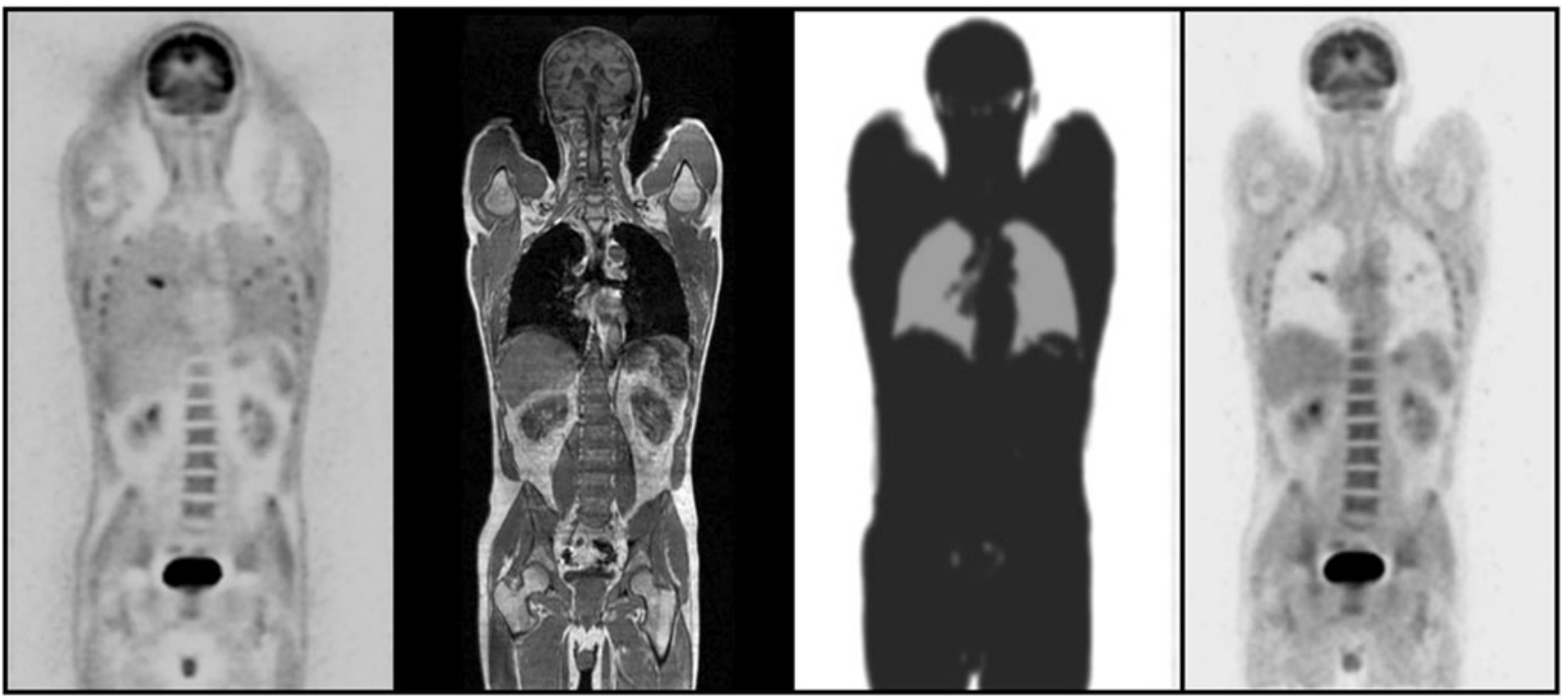

Uncorrected PET

Whole body MRI

Attenuation Mask

Corrected PET
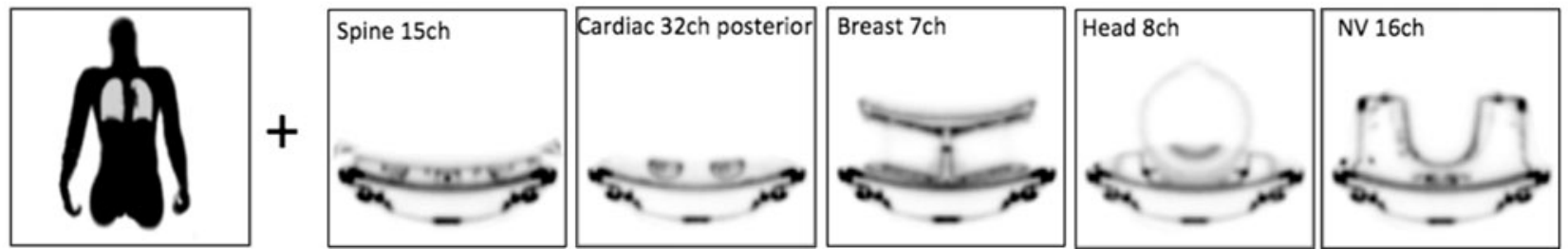

Fig. 2 Attenuation correction of PET images is based on a 3D whole-body attenuation mask calculated from a segmentation of a whole-body MRI and assigning different attenuation factors to different tissue, such soft tissue, air and external contour of the body.

We can also anticipate that the development of new PET tracers will increase the potential of hybrid PET/MR imaging protocols. One of the forthcoming tracers is ${ }^{18} \mathrm{~F}$ labeled sodium fluoride $(\mathrm{NaF})$, which is being used for detection of bone metastases. Other tracers are starting to be widely adopted in clinical practice today, such as ${ }^{18} \mathrm{~F}$ fluorocholine for prostate cancers, ${ }^{18} \mathrm{~F}$-DOPA for the detection of endocrine tumors, as well as new tracers for the detection of myocardial perfusion and evaluation of the extent of amyloid plaques in neurodegenerative diseases. Numerous other tracers are being developed and tested in clinical trials and will soon be available for clinical use. With the superiority of MR over CT for detection of bone lesions and differentiation of malignant versus benign lesions, PET/MR can be expected to perform better than $\mathrm{PET} / \mathrm{CT}$ in this particular domain.

The primary clinical applications of PET/MR today are in oncology, but there are other emerging applications in cardiology and in neurodegenerative diseases. MRI is now widely used in cardiology for the detection of ischemic disease, myocardial viability and cardiac function. The
In addition to individually calculated attenuation maps, pre-defined attenuation maps of surface coils used for different diagnostic sequences are added to the whole-body mask (lower row)

added value and complementarity of PET in providing greater sensitivity and a better quantitative analysis of myocardial perfusion, as well as in identifying residual viable myocardium in scar tissue, could constitute good clinical justifications for combined PET/MR studies. Recent preliminary studies have also shown that combined PET/MR findings could help differentiate active and potentially dangerous arterial plaques from stable, chronic lesions of the vascular wall.

Brain imaging for acute as well as chronic disease often consists of a combination of multiple imaging modalities such as PET and MR. However, thanks to the rigidity of the head and the easily identifiable skull structures, softwarebased image registration techniques provide adequate fusion of different imaging modalities for accurate localization of different brain structures from each modality. Hybrid PET/MR could, however, become more widely available and more widely used in particular to reduce imaging time and for the convenience of the patient, who is spared the need to undergo separate studies on different scanners. Integrated PET/MR systems allowing 
simultaneous acquisition of both modalities could also become valuable for functional studies and in the assessment of some cognitive mechanisms.

\section{Discussion and conclusion}

While the added value of hybrid PET/CT over the two modalities acquired separately has been well established clinically [14-16], the expected added value of hybrid PET/MR remains to be demonstrated [17-19]. While no "killer application" for PET/MR over other imaging modalities has yet been clearly identified, there is already some evidence that this hybrid modality can, today, be advantageous in patients who require both PET and MRI in their workup. Additional clinically relevant information can be gathered from MR imaging in domains where, today, MR can be complementary to PET/CT. This is particularly true of prostate cancer [20] and breast cancer $[21,22]$, as well as head and neck cancers where anatomical registration of studies acquired separately is difficult and sometimes misleading.

Unlike CT scans limited by the amount of radiation, MR offers the advantage that additional segmental scans can be acquired with higher resolution and better tissue differentiation on specific body areas (see examples in Fig. 3). Additional relevant information and tissue characterization can also be obtained in selected cases by intravenous injection of MR contrast material.

A limiting factor that must be addressed before hybrid PET/MR can be used in clinical routine is the total duration of MR imaging protocols. Adding PET scanning time to full diagnostic clinical MRI protocols can result in studies that are significantly longer than the standard PET/CT study. In hybrid PET/MRI, it is necessary to shorten the MR acquisition protocol to limit the duration of the study to a clinically acceptable time. While advanced MR imaging sequences can provide relevant diagnostic information, they cannot be applied to whole-body imaging. Specific imaging sequences will have to be restricted to the organs and body sections that benefit the most from such imaging protocols. Our preliminary experience shows that whole-body PET imaging with TOF technology can be achieved in under $15 \mathrm{~min}$; adding this to a whole-body attenuation and localization MRI scan acquired in under 5 min results in a combined PET/MR scan acquired in $<20$ min. Most clinical protocols would require between two and five additional MRI series with dedicated (a) Head \& Neck

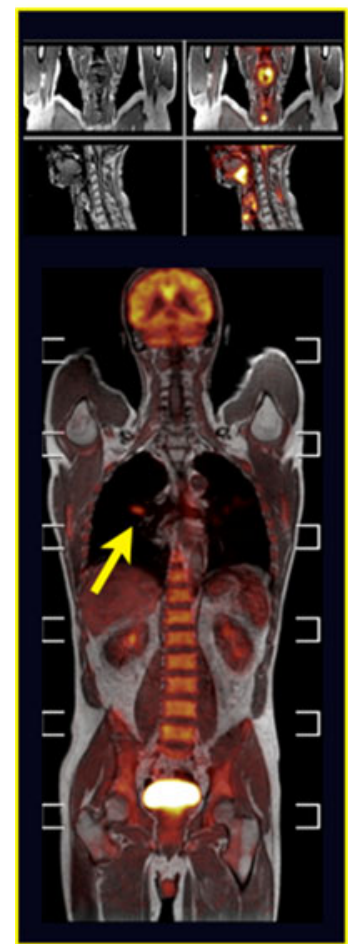

(b) Breast

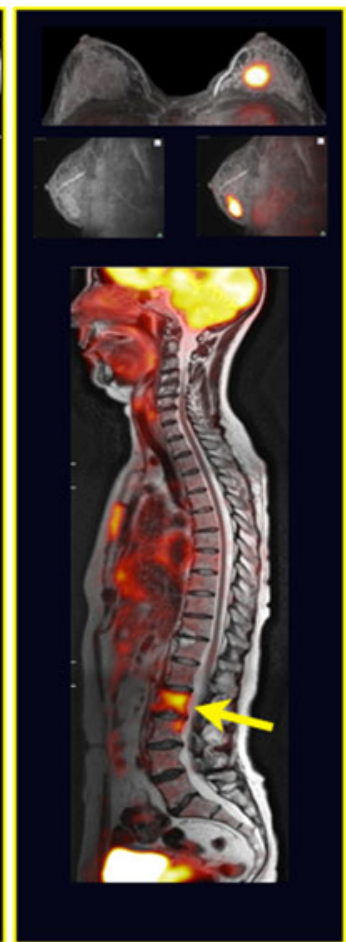

(c) Prostate
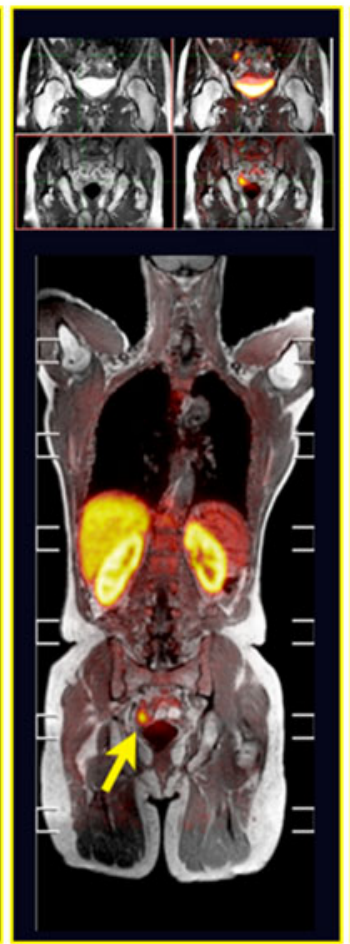

(d) Sarcoma

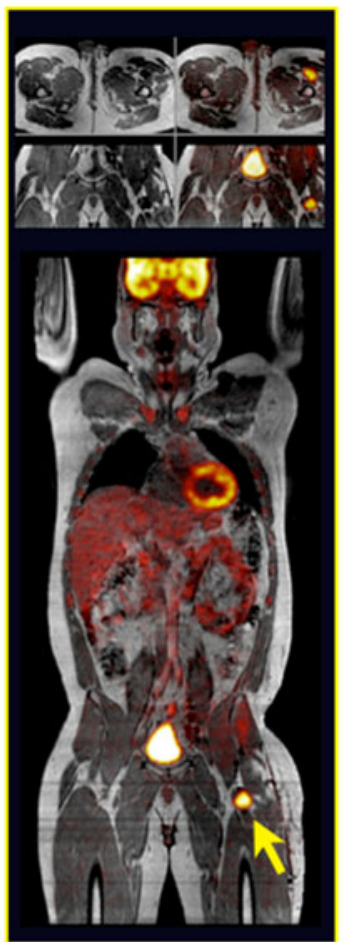

(e) Pelvis

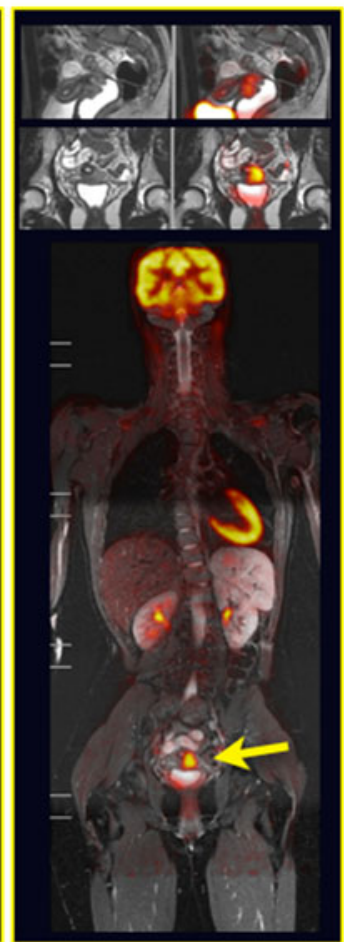

Fig. 3 Examples of applications of diagnostic PET/MR scans in oncology, with high-resolution diagnostic MR protocols (upper row) and whole-body scans (lower row). From left to right: a head and neck cancer with focal uptake of the right hilar region consistent with a lung metastases, b breast cancer with lumbar spine metastases, c recurrence of a prostate cancer with focal uptake in the prostate area, $\mathbf{d}$ sarcoma of the left thigh, and e cervical cancer of the uterus 
protocols on specific organs or body sections. These additional sequences can also be completed in under 30 min, making it possible to complete a diagnostic PET/ MR scan within an hour. While this study is longer than a standard diagnostic PET/CT study, it is still shorter than the total time that a PET/CT and an MRI study would take today, performed on two separate scanners at different times. This clearly benefits those patients who need both PET/CT and MR studies in their clinical workup. The use of PET/MR in clinical practice is expected to increase further with the emergence of new radiolabeled tracers.

Another factor that favors MRI over CT for hybrid imaging devices is the reduction in radiation exposure, as it eliminates the ionizing radiation of CT. Although radiation exposure may not be a major issue in elderly patients or patients under palliative treatment for cancer, reduced radiation exposure of PET/MRI compared with PET/CT is of interest in non-oncological patients and in younger patients with a potentially curable disease [23, 24].

\section{Conflict of interest None}

\section{References}

1. Collins CD (2007) PET/CT in oncology: for which tumours is it the reference standard? Cancer Imaging 7(Spec No A):S77-S87

2. Lonsdale MN, Beyer T (2010) Dual-modality PET/CT instrumentation-today and tomorrow. Eur J Radiol 73:452-460

3. Antoch G, Saoudi N, Kuehl H et al (2004) Accuracy of wholebody dual-modality fluorine-18-2-fluoro-2-deoxy-D-glucose positron emission tomography and computed tomography (FDGPET/CT) for tumor staging in solid tumors: comparison with CT and PET. J Clin Oncol 22:4357-4368

4. Bar-Shalom R, Yefremov N, Guralnik L et al (2003) Clinical performance of PET/CT in evaluation of cancer: additional value for diagnostic imaging and patient management. J Nucl Med 44:1200-1209

5. Antoch G, Bockisch A (2009) Combined PET/MRI: a new dimension in whole-body oncology imaging? Eur J Nucl Med Mol Imaging 36(Suppl 1):S113-S120

6. Liang X, Congxin W, Yi Z, Jinwen H, Chuanfu L (2011) Wholebody diffusion-weighted MR imaging with fat suppression by using STIR: clinical utility for detection of primary malignancies in patients initially presented with metastasis. Eur $\mathrm{J}$ Radiol 79:359-362

7. Chen W, Jian W, Li HT et al (2010) Whole-body diffusionweighted imaging vs. FDG-PET for the detection of non-smallcell lung cancer. How do they measure up? Magn Reson Imaging 28:613-620

8. Shao Y, Cherry SR, Farahani K et al (1997) Simultaneous PET and MR imaging. Phys Med Biol 42:1965-1970
9. Beyer T, Pichler B (2009) A decade of combined imaging: from a PET attached to a CT to a PET inside an MR. Eur J Nucl Med Mol Imaging 36(Suppl 1):S1-S2

10. Beyer T, Weigert M, Quick HH et al (2008) MR-based attenuation correction for torso-PET/MR imaging: pitfalls in mapping MR to CT data. Eur J Nucl Med Mol Imaging 35:1142-1146

11. Hofmann M, Pichler B, Scholkopf B, Beyer T (2009) Towards quantitative PET/MRI: a review of MR-based attenuation correction techniques. Eur J Nucl Med Mol Imaging 36(Suppl 1):S93-S104

12. Hu Z, Ojha N, Renisch S et al (2009) MR-based attenuation correction for a whole-body sequential PET/MR system. In: IEEE nuclear science symposium conference record, Orlando 2009, pp 3508-3512

13. Delso G, Ziegler S (2009) PET/MRI system design. Eur J Nucl Med Mol Imaging 36(Suppl 1):S86-S92

14. Fletcher JW, Kymes SM, Gould M et al (2008) A comparison of the diagnostic accuracy of ${ }^{18}$ F-FDG PET and CT in the characterization of solitary pulmonary nodules. J Nucl Med 49:179-185

15. Yang W, Fu Z, Yu J et al (2008) Value of PET/CT versus enhanced CT for locoregional lymph nodes in non-small cell lung cancer. Lung Cancer 61:35-43

16. Czernin J, Allen-Auerbach M, Schelbert HR (2007) Improvements in cancer staging with PET/CT: literature-based evidence as of September 2006. J Nucl Med 48(Suppl 1):78S-88S

17. Platzek I, Zastrow S, Deppe PE et al (2010) Whole-body MRI in follow-up of patients with renal cell carcinoma. Acta Radiol 51:581-589

18. Venkitaraman R, Cook GJ, Dearnaley DP et al (2009) Wholebody magnetic resonance imaging in the detection of skeletal metastases in patients with prostate cancer. J Med Imaging Radiat Oncol 53:241-247

19. Schmidt GP, Reiser MF, Baur-Melnyk A (2009) Whole-body MRI for the staging and follow-up of patients with metastasis. Eur J Radiol 70:393-400

20. Van Goethem M, Tjalma W, Schelfout K, Verslegers I, Biltjes I, Parizel P (2006) Magnetic resonance imaging in breast cancer. Eur J Surg Oncol 32:901-910

21. Heusner TA, Kuemmel S, Koeninger A et al (2010) Diagnostic value of diffusion-weighted magnetic resonance imaging (DWI) compared to FDG PET/CT for whole-body breast cancer staging. Eur J Nucl Med Mol Imaging 37:1077-1086

22. Heusner TA, Kuemmel S, Umutlu L et al (2008) Breast cancer staging in a single session: whole-body PET/CT mammography. J Nucl Med 49:1215-1222

23. Kleis M, Daldrup-Link H, Matthay K et al (2009) Diagnostic value of PET/CT for the staging and restaging of pediatric tumors. Eur J Nucl Med Mol Imaging 36:23-36

24. Punwani S, Taylor SA, Bainbridge A et al (2010) Pediatric and adolescent lymphoma: comparison of whole-body STIR halfFourier RARE MR imaging with an enhanced PET/CT reference for initial staging. Radiology 255:182-190

25. Bao A, Goins B, Dodd GD 3rd et al (2008) Real-time iterative monitoring of radiofrequency ablation tumor therapy with ${ }^{15} \mathrm{O}$ water PET imaging. J Nucl Med 49:1723-1729 